\section{Nanoscale Conformation and Compressibility of Cartilage Aggrecan Using Microcontact Printing and Atomic Force Microscopy}

\author{
Delphine Dean, ${ }^{\dagger}$ Lin Han, ${ }^{\ddagger}$ Christine Ortiz, ${ }^{\ddagger}$ and \\ Alan J. Grodzinsky*,†,
}

Department of Electrical Engineering and Computer Science, Department of Materials Science and Engineering, and Biological Engineering Division, Massachusetts Institute of Technology, 77 Massachusetts Avenue, Cambridge, Massachusetts 02139

\section{Received November 17, 2004}

Revised Manuscript Received March 25, 2005

Introduction. The nanomechanical behavior of cartilage extracellular matrix (ECM) macromolecules has received increasing attention ${ }^{1-4}$ since ECM degradation leads to tissue and joint dysfunction with age and arthritis. ${ }^{5}$ For example, loss of the branched proteoglycan, aggrecan, and its highly negatively charged constituent glycosaminoglycan (GAG) polymer chains (Figure 1) significantly reduces cartilage's compressive stiffness. ${ }^{5}$ The nanoscale force between opposing endgrafted GAG layers (a system that mimics local molecular responses to tissue deformations) has been measured over a broad range of $\mathrm{pH}$ and ionic strength (IS) solution conditions ${ }^{2}$ and was well described by a Poisson-Boltzmann-based, electrostatic double layer (EDL) "charged rod" model. ${ }^{6}$ In vivo, however, GAGs are covalently linked to a core protein, creating a dense cylindrical "bottle-brush" architecture, thus motivating studies on intact aggrecan, which is a closer-to-physiological model system. Here, we probe the conformation and compressibility of an aggrecan-functionalized surface by combining the techniques of microcontact printing $\left(\mu \mathrm{CP}^{7,8}\right)$ and contact mode atomic force microscopy (AFM) (Figure 1). $\mu \mathrm{CP}$ enabled the creation of a patterned surface with densely packed, chemically endgrafted aggrecan confined to well-defined micrometersized surface areas and a hydroxyl-terminated selfassembling monolayer (OH-SAM) filling the rest of the surface area. By AFM imaging over boundaries between aggrecan and $\mathrm{OH}-\mathrm{SAM}$ regions, the height and, hence, the deformation of the aggrecan were directly measured as a function of aqueous bath conditions (IS $=0.001-1$ $\mathrm{M}, \mathrm{pH}=3-7)$ and normal compressive load $(0-40 \mathrm{nN})$.

Experimental Section. Fetal bovine aggrecan ${ }^{9}(0.5$ $\mathrm{mg} / \mathrm{mL}$ ) was solubilized in deionized (DI) water with 1 $\mu \mathrm{M}$ dithiobis(sulfosuccinimidyl propionate) (Pierce \#21578), which can react with amine groups at the $\mathrm{N}$-terminal and lysines at the $\mathrm{N}$ - and $\mathrm{C}$-termini of the core protein, thus forming disulfide bonds. These disulfides were reduced to thiols by reacting with dithiothreitol (Pierce \#150460) in $0.1 \mathrm{mM}$ aqueous solution for $1 \mathrm{~h}$. Excess reactants were removed by spinning at $3500 \mathrm{rpm}$ overnight with a centrifugal filter (Centricon, Millipore, $10 \mathrm{kDa}$ cutoff). OH-aggrecan patterned substrates were made by $\mu \mathrm{CP}^{7}$ of $4 \mathrm{mM} 11$-mercaptounde-

\footnotetext{
† Department of Electrical Engineering and Computer Science.

Department of Materials Science and Engineering.

$\S$ Biological Engineering Division.

* Corresponding author. E-mail: alg@mit.edu.
}

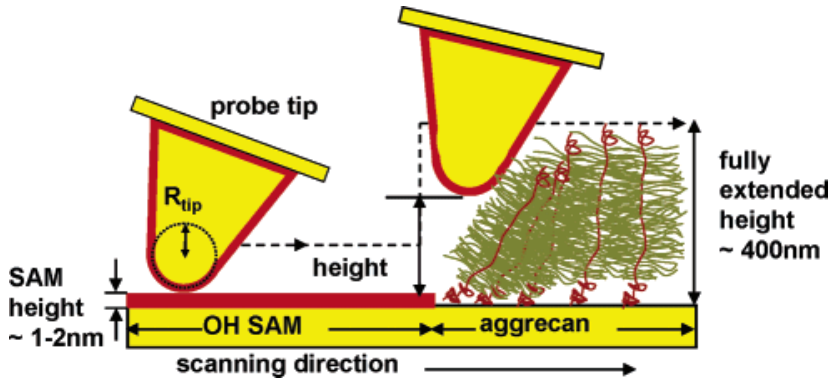

Figure 1. Illustration of AFM contact mode imaging on aggrecan and hydroxyl-terminated SAM patterned surface $\left(R_{\text {tip }}\right.$ $\sim 50 \mathrm{~nm}$, aggrecan molecules spaced $\sim 20 \mathrm{~nm}$ apart, contour length $\sim 400 \mathrm{~nm}$, side chain (mostly GAG) contour length $\sim 40$ $\mathrm{nm}$, side chains spaced $\sim 2-4 \mathrm{~nm}$ apart).

canol, $\mathrm{HS}\left(\mathrm{CH}_{2}\right)_{11} \mathrm{OH}$ (Aldrich), in ethanol on a freshly cleaned $1 \mathrm{~cm} \times 1 \mathrm{~cm}$ gold-coated substrate ${ }^{10}$ for $30 \mathrm{~s}$ using a hexagonal patterned poly(dimethylsiloxane) (PDMS) stamp (hexagon side length $=15 \mu \mathrm{m}$ and spacing $=15 \mu \mathrm{m})$ followed by incubation in $50 \mu \mathrm{L}$ of 1 $\mathrm{mg} / \mathrm{mL}$ thiol-terminated aggrecan solution for $48 \mathrm{~h}$. Hence, the aggrecan was located within the hexagons and the $\mathrm{OH}-\mathrm{SAM}$ outside the hexagons. $\mathrm{OH}$-functionalized AFM gold-coated probe tips (Digital Instruments, $k \sim 0.06 \mathrm{~N} / \mathrm{m}$, tip radius $\sim 50 \mathrm{~nm}$ ) were prepared by immersion in $4 \mathrm{mM}$ 11-mercaptoundecanol for $48 \mathrm{~h}$ to minimize electrostatic interactions between the probe tip and aggrecan layer.

Aggrecan height in aqueous solution was measured as the relative height between the OH-SAM (negligible thickness $\sim 1-2 \mathrm{~nm}$ ) and aggrecan (Figure 1) regions under different compressive normal forces using a Multimode Nanoscope IV AFM (Digital Instruments) in contact mode in fluid at a scanning frequency of $1 \mathrm{~Hz}$. Solutions were $0.001-1 \mathrm{M} \mathrm{NaCl}$ in Milli-Q water $(\mathrm{pH}$ $\sim 5.6$ ) and $0.01 \mathrm{M}$ phosphate buffered saline (PBS) at $\mathrm{pH}=3,7$, and 10 and $0.154 \mathrm{M} \mathrm{PBS}$ at $\mathrm{pH}=7.4$. After AFM experiments were completed, aggrecan grafting density on the surface was assessed by removal of the aggrecan via boiling the substrate in DI water for 60 min. The residual solution was lyophilized and redissolved in $50 \mu \mathrm{L}$ of DI water. A dimethylmethylene blue (DMMB) dye binding assay ${ }^{11}$ yielded a total aggrecan surface density equivalent to $\sim 10.1 \pm 1.4 \mathrm{mg} / \mathrm{m}^{2}$, or one aggrecan molecule per $\sim 20 \mathrm{~nm} \times 20 \mathrm{~nm}$. The GAGs from this fetal bovine aggrecan have contour lengths of $\sim 40 \mathrm{~nm}^{9}$

Results and Discussion. Aggrecan height was found to be nonhysteretic (independent of previous solvent conditions and loading history) and decreased markedly with increasing IS from 0.001 to $1 \mathrm{M}$ under constant normal compressive load (Figure 2) and with increasing normal load at constant IS (Figure 3a). The maximum height, $\sim 310 \mathrm{~nm}$ in $0.001 \mathrm{M} \mathrm{NaCl}$ and $\sim 0 \mathrm{nN}$ normal force, was $\sim 78 \%$ of the known contour length of the aggrecan used $(\sim 400 \pm 60 \mathrm{~nm}) .{ }^{9}$ The strong dependence of aggrecan height on IS indicates that electrostatic interactions are a critical determinant of the nanomechanical behavior of aggrecan. The carboxyl and sulfate groups on the CS-GAG are ionized at all bath $\mathrm{pH}$ and IS of Figure $3 .^{10}$ The electrostatic interaction distance in monovalent electrolyte solutions is determined by Debye length, $\kappa^{-1}=\sqrt{\epsilon_{\mathrm{w}} R T / 2 F^{2} C_{0}}$ (where $\epsilon_{\mathrm{w}}$ is the 


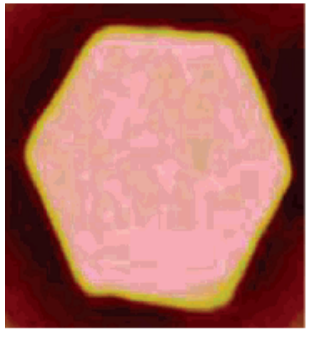

(a) $0.001 \mathrm{M}$

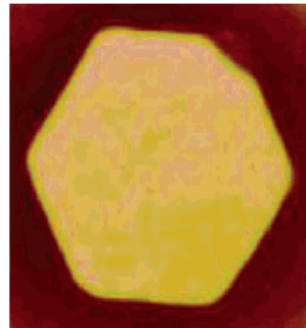

(b) $0.01 \mathrm{M}$

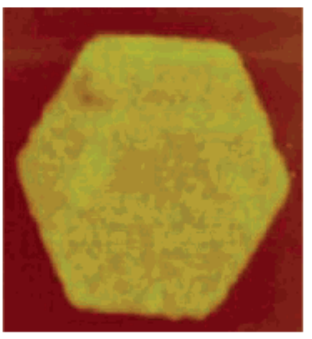

(c) $0.1 \mathrm{M}$

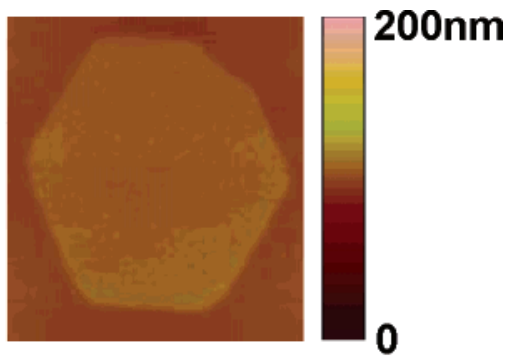

(d) $1.0 \mathrm{M}$

Figure 2. AFM contact mode height images of $\mu \mathrm{CP}$ surfaces with aggrecan inside and a hydroxyl-terminated SAM outside the hexagons (20 $\mu \mathrm{m}$ scan; $3 \mathrm{nN}$ applied normal force) at different ionic strengths $(0.001-1 \mathrm{M} \mathrm{NaCl}, \mathrm{pH}=5.6$ ).
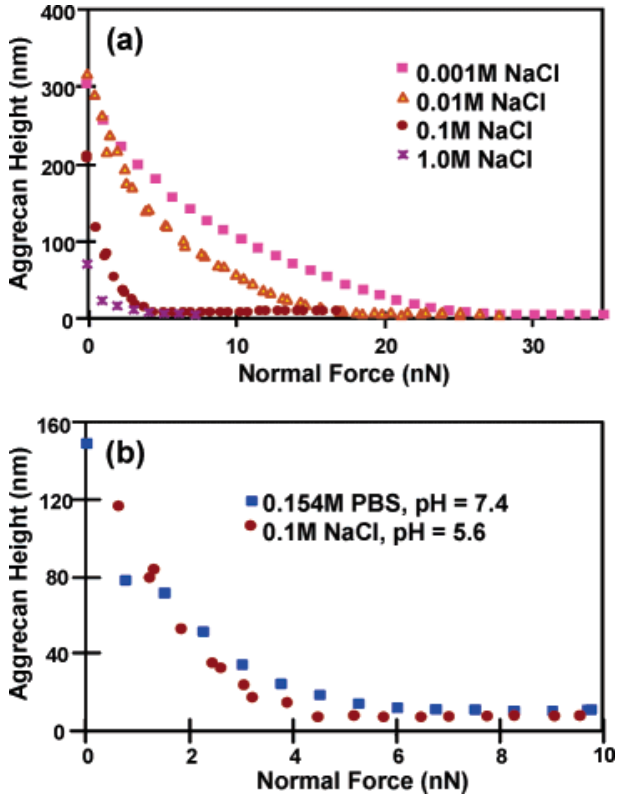

Figure 3. Aggrecan height as a function of normal force and ionic strengths: (a) $0.001-1.0 \mathrm{M} \mathrm{NaCl}, \mathrm{pH}=5.6$ and (b) 0.154 $\mathrm{M}$, phosphate buffered saline (PBS), $\mathrm{pH}=7.4$ and $0.1 \mathrm{M} \mathrm{NaCl}$, $\mathrm{pH}=5.6$ (standard deviations were smaller than the size of data points).

permittivity $\left(6.92 \times 10^{-10} \mathrm{C} /\left(\mathrm{N} \mathrm{m}^{2}\right)\right), F$ the Faraday constant (96500 C/mol), $R$ the universal gas constant, $T$ the absolute temperature $(298 \mathrm{~K})$, and $C_{0}$ the bath IS). At low IS $(<0.1 \mathrm{M} \mathrm{NaCl}), \kappa^{-1}$ is greater than the spacing between GAG chains on aggrecan $(\sim 2-4 \mathrm{~nm})$, resulting in large electrostatic repulsion forces between neighboring and adjacent GAG chains. These forces facilitate extension of the aggrecan monomers from the gold substrate, giving an uncompressed aggrecan height that is close to the contour length of aggrecan. As the IS was increased, electrostatic repulsion forces are shielded, and the height of the aggrecan decreases. At $1 \mathrm{M} \mathrm{NaCl}$, the majority of the interchain electrostatic repulsion has been screened out, and the resulting uncompressed height of the aggrecan layer $(\sim 100 \mathrm{~nm})$ is determined mostly by steric effects as well as some intrachain electrostatic repulsion along the CS-GAGs due to the close spacing of charge groups along the chains $(\sim 0.6 \mathrm{~nm})$.

For all bath IS, there was an initial steep decrease in height with increasing normal force followed by a plateauing of height at higher forces, corresponding to an "incompressible height." With decreasing IS, greater normal force was needed to attain this incompressible height. Although the initial height of the aggrecan at $\sim 0 \mathrm{nN}$ force changed greatly with ionic strength, the incompressible height at high normal force $(>30 \mathrm{nN})$ was $\sim 5 \mathrm{~nm}$ for all IS conditions (Figure $3 \mathrm{a}$ ). In $0.001 \mathrm{M}$ $\mathrm{NaCl}$, the aggrecan appeared stiffest (i.e., the height vs normal force curve had the smallest slope) due to high electrostatic repulsion forces. Although aggrecan height at $\sim 0 \mathrm{nN}$ was similar in 0.01 and $0.001 \mathrm{M} \mathrm{NaCl}$, the aggrecan height decayed more steeply with increasing normal force in $0.01 \mathrm{M} \mathrm{NaCl}$. For IS $>0.01 \mathrm{M}$, the aggrecan became more compliant with increasing IS. By 1.0 $\mathrm{M} \mathrm{NaCl}$, when the inter-GAG chain electrostatic interactions are almost completely shielded and nonelectrostatic effects dominate, the aggrecan height decayed rapidly with normal force, reaching its incompressible height at the relatively low force of $\sim 5 \mathrm{nN}$.

Aggrecan height measured in $0.01 \mathrm{M} \mathrm{PBS}$ at $\mathrm{pH}=3$, 7 , and 10 was not significantly different from the height in $0.01 \mathrm{M} \mathrm{NaCl}$ at $\mathrm{pH}=5.6$ for all normal forces used (data not shown). Furthermore, aggrecan height in physiological conditions $(0.154 \mathrm{M}$ PBS at $\mathrm{pH}=7.4)$ was not significantly different from the height in $0.1 \mathrm{M} \mathrm{NaCl}$ at $\mathrm{pH}=5.6$ (Figure 3b). Although the degree of protonation of carboxyl groups at $\mathrm{pH} 3$ is different from that at $\mathrm{pH} 7,{ }^{12}$ the sulfate groups would not be significantly affected. ${ }^{13}$ Even at $\mathrm{pH}=3$, when the total aggrecan fixed charge is approximately half that at $\mathrm{pH}$ 7 , there are still very strong GAG-GAG electrostatic repulsion forces at $0.01 \mathrm{M}$ IS from the sulfate groups alone that cause the aggrecan to have the same height. This is consistent with the observation that $\kappa^{-1}(0.01 \mathrm{M})$ $\sim 3 \mathrm{~nm}$ is on the order of the inter-GAG chain spacing along the aggrecan core protein. In contrast, the height of a sparsely populated CS-GAG brush (measured by ellipsometry $)^{2}$ was found previously to decrease from $\mathrm{pH}$ $=5$ to 3 at $0.01 \mathrm{M}$ IS. However, the GAG-GAG spacing in that system $(6.5 \mathrm{~nm})$ was $\sim 3-4$ times greater than that within the aggrecan brush used here. Finally, the aggrecan height vs normal force behavior at $0.1 \mathrm{M} \mathrm{NaCl}$, pH 5.4 (Figure 3b), was similar to that under the physiologic buffer conditions (IS $=0.154 \mathrm{M}, \mathrm{pH}$ 7.4). The volumetric density of aggrecan on the surface was calculated using the measured surface density $(\sim 10.1$ $\mathrm{mg} / \mathrm{m}^{2}$ ) and the height data at physiological solution conditions (IS $=0.154 \mathrm{M}, \mathrm{pH} 7.4$, Figure $3 \mathrm{~b}$ ) at $\sim 0 \mathrm{nN}$ force (corresponding to a $150 \mathrm{~nm}$ aggrecan height) and yielded $\sim 56 \mathrm{mg} / \mathrm{mL}$, which is within the known range of aggrecan concentrations in normal articular cartilage under free swelling conditions $(20-80 \mathrm{mg} / \mathrm{mL}) .^{5}$

The $\mu$ CP-AFM method does have some limitations. First, since a small tare force of up to $\sim 200 \mathrm{pN}$ is necessary to attain stable feedback for AFM imaging, nonnegligible compressions may result in underestimations of the equilibrium polymer heights at $\sim 0 \mathrm{nN}$. Second, the resolution of this technique depends on the 
surface roughness of the substrate. For thinner $(<5 \mathrm{~nm})$ polymer layers, the gold substrate used here may be replaced by mica to achieve better resolution of small height differences between the inside and the outside of the patterned region. Conversely, the $\mu \mathrm{CP}-\mathrm{AFM}$ method has several advantages for the measurement of polymer thicknesses compared to other approaches such as the scratch method, ${ }^{14}$ ellipsometry, ${ }^{15}$ neutron reflectivity, ${ }^{15}$ etc. The $\mu \mathrm{CP}$-AFM method offers a more well-defined chemical system and better resolution $(\sim \mathrm{nm})$ than the often-used "scratch method"14 and provides a means to directly measure the polymer height in a variety of solvent conditions and under a range of normal forces. This is not possible with more conventional techniques such as ellipsometry. ${ }^{16}$ By measuring the height under different applied normal forces, additional information can be gathered about the nanomechanical properties of the polymer layer.

In this study, the aggrecan layer height and compressibility of aggrecan were observed simultaneously, which enables a direct estimate of the stiffness of the layer. Additionally, other mechanical properties can be investigated via $\mu \mathrm{CP}$. By using a colloidal tip having a radius on the micrometer scale, the polymer layer can be sandwiched between surfaces with a much larger radius of curvature, resulting in a simpler boundary condition for measurement of mechanical moduli than that of a sharp pyramidal tip. Measurement of polymer height vs normal force can be also be combined with normal force vs separation distance curves, giving a more complete description of the mechanical behavior of the layer at both small and large loads. Using lateral force microscopy, the shear behavior of polymer layers can be also be studied with brush height obtained simultaneously; chemical grafting of the polymer may offer advantages over physical attachment for investigating nanomechanical behavior. ${ }^{17}$
Acknowledgment. The authors thank Cheolmin Park for donating the PDMS stamp used in these experiments. Funding was from NIH Grant AR33236, NSF-NIRT 0403903, and a Whitaker Foundation Fellowship (D.D.).

\section{References and Notes}

(1) Liu, X.; Noble, P. C.; Luo, Z. P. Biochem. Biophys. Res. Commun. 2003, 307, 338-341.

(2) Seog, J.; Dean, D.; Rolauffs, B.; Wu, T.; Genzer, J.; Plaas, A. H. K.; Grodzinsky, A.; Ortiz, C. J. Biomech., in press.

(3) Stolz, M.; Raiteri, R.; Daniels, A. U.; VanLandingham, M. R.; Baschong, W.; Aebi, U. Biophys. J. 2004, 86, 3269-3283.

(4) Sun, Y. L.; Luo, Z. P.; Fertala, A.; An, K. N. Biochem. Biophys. Res. Commun. 2002, 295, 382-386.

(5) Maroudas, A. In Adult Articular Cartilage; Freeman, M. A. R., Ed.; Pitman: England, 1979; pp 215-290.

(6) Dean, D.; Seog, J.; Ortiz, C.; Grodzinsky, A. Langmuir 2003 $19,5526-5539$.

(7) Wilbur, J. L.; Kumar, A.; Kim, E.; Whitesides, G. M. Adv. Mater. 1994, 6, 600-604.

(8) Braun, H. G.; Meyer, E. Macromol. Rapid Commun. 1999, $20,325-327$.

(9) Ng, L.; Grodzinsky, A. J.; Patwari, P.; Sandy, J.; Plaas, A.; Ortiz, C. J. Struct. Biol. 2003, 143, 242-257.

(10) Seog, J.; Dean, D.; Plaas, A. H. K.; Wong-Palms, S.; Grodzinsky, A. J.; Ortiz, C. Macromolecules 2002, 35, 56015615.

(11) Farndale, R. W.; Buttle, D. J.; Barrett, A. J. Biochim. Biophys. Acta 1986, 883, 173-177.

(12) Freeman, W. D. S. C.; Maroudas, A. Ann. Rheum. Dis. 1975 , $34,44-45$.

(13) Kuettner, K.; Lindenbaum, A. Biochim. Biophys. Acta 1965, $101,223-225$

(14) Yamamoto, S.; Ejaz, M.; Tsujii, Y.; Matsumoto, M.; Fukuda, T. Macromolecules 2000, 33, 5602-5607.

(15) Tran, Y.; Auroy, P.; Lee, L.-T. Macromolecules 1999, 32, 8952-8964.

(16) Biesalski, M.; Rühe, J. Macromolecules 1999, 32, 23092316.

(17) Raviv, U.; Giasson, S.; Kampf, N.; Gohy, J. F.; Jerome, R.; Klein, J. Nature (London) 2003, 425, 163-165.

MA047626K 